\section{An evaluation of class effect}

The results of Zhou's retrospective observational study ${ }^{1}$ conflict with the evidence obtained from well-designed clinical trials. Retrospective analyses of administrative databases may indicate an association between 2 variables, but one must not infer that a causal relation exists.

Limitations in the study design complicate interpretation of the results. Equivalence trials are designed to confirm the absence of a meaningful difference between treatments where a margin of clinical equivalence is prespecified, which was not the case here. If equivalence trials are not designed and analyzed appropriately, they often have intrinsic biases tending toward the conclusion of no difference. ${ }^{2,3}$

Despite adjustments for many confounding variables, the study did not capture a key independent risk factor that affects baseline cardiovascular risk, namely total cholesterol or lowdensity lipoprotein cholesterol blood concentration. ${ }^{4}$ Finally, the rate of switches for non-atorvastatin users was high, resulting in "contamination" of other statin groups with atorvastatin users. This was not appropriately accounted for in the analyses. Switching may occur not only because of worsening clinical status, but also because of higher baseline cholesterol, which confers a higher cardiovascular risk.

Zhou highlights the care gap observed between 1997 and 2001: $67 \%$ of elderly subjects did not receive lipidlowering therapy after myocardial infarction, and most of the remaining patients received low starting doses of statins. It is encouraging, however, that persistence rates were high, which is important in optimizing care after myocardial infarction.

\section{Bernard Prigent}

Medical Director and Vice-President Pfizer Canada

Kirkland, Que.

\section{REFERENCES}

I. Zhou Z, Rahme E, Abrahamowicz M, et al. Effectiveness of sttains for secondary prevention in elderly patients after acute myocardial infarction: an evaluation of class effect. CMAJ 2005;172(9):II87-94.

2. Greene WL, Concato J, Feinstein AR. Claims of equivalence in medical research: Are they supported by the evidence? Ann Intern Med 2000; I32: 715-22.

3. Jones B, Javis P, Lewis JA, et al. Trials to assess equivalence: the importance of rigorous methods. BMJ I996;313:36-9.

4. Genest J, Frohlich J, Fodor G, et al. Recommendations for the management of dyslipidemia and the prevention of cardiovascular disease: 2003 update. CMAJ 2003;169(9):92I-4.

DOI:I0.I503/cmaj.I050I29

\section{[Three of the authors respond:]}

Complementary roles of observational studies and randomized controlled trials (RCTs) have been recognized. ${ }^{1}$ Results from RCTs comparing statins head-to-head for long-term cardiovascular prevention are, in fact, very few. The Pravastatin or Atorvastatin Evaluation and Infection Therapy (PROVE-IT) trial $^{2}$ and the recent Incremental Decrease in Endpoints through Aggressive Lipid Lowering (IDEAL) trial ${ }^{3}$ compared 2 different statin regimens with a focus on comparing intensive versus moderate cholesterol-lowering therapy, rather than the different statins per se. The latter, comparing high-dose atorvastatin versus usual-dose simvastatin, failed to achieve significance in the primary endpoint of major coronary events. ${ }^{3}$ Compared to subjects enrolled in clinical trials, the present observational study ${ }^{4}$ evaluated the effectiveness of statins in all patients $\geq 65$ year of age with a diverse risk profile. Thus, our study provided evidence in real-world practice.

Our study was not an equivalence trial but a study of the effectiveness of the different statins prescribed to the population at large. Posterior power calculation is theoretically less meaningful in the observational study setting, where patients from the 3 most populated provinces in Canada were included. ${ }^{4}$ The confidence intervals around the point estimate of I.O we observed were quite narrow and suggested a class effect of statins.

Missing patient cholesterol information represents a limitation in the study, especially for the study of statin dose effect. However, there is no obvious reason to believe that cholesterol levels were significantly different across 5 statin groups. In our study population, the median dose used across statins was comparable, and in our analyses we adjusted for dose equivalence. There is a possibility that switching to atorvastatin caused "contamination." However, as reported in our sensitivity analysis, we found that patients who switched to atorvastatin or to other statins had similar risk profiles (as measured by similar rates of cardiac outcomes and medication use). Additionally, similar results were obtained when we kept patients who switched in the analysis (intention to treat) or removed them. Thus we believe that the clinical risk was similar across groups.

We agree that higher doses of statins should be used to attain the lowest low-density lipoprotein target possible, regardless of which statin is prescribed. ${ }^{5}$

\section{Zheng Zhou \\ Elham Rahme \\ Louise Pilote \\ Divisions of Clinical Epidemiology \\ and Internal Medicine \\ Montreal General Hospital \\ McGill University Health Centre \\ Montréal, Que.}

\section{REFERENCE}

I. Black N. Why we need observational studies to evaluate the effectiveness of health care. $B M J$ I 996 ; 312:1215-8.

2. Cannon $\mathrm{CP}$, Braunwald $\mathrm{E}, \mathrm{McC}$ abe $\mathrm{CH}$, et al. Intensive versus moderate lipid lowering with statins after acute coronary syndromes. $N$ Engl J Med 2004; 350:I495-504.

3. Pedersen TR, Faergeman O, Kastelein JJ, et al. High-dose atorvastatin vs usual-dose simvastatin for secondary prevention after myocardial infarction: the IDEAL study: a randomized controlled trial. JAMA 2005;294:2437-45.

4. Zhou Z, Rahme E, Abrahamowicz M, et al. Effectiveness of sttains for secondary prevention in elderly patients after acute myocardial infarction: an evaluation of class effect. CMAJ 2005;172(9):1187-94.

5. Sacks FM. High-intensity statin treatment for coronary heart disease. JAMA 2004;291:II32-4.

Competing interests: None declared.

DOI:Io.I503/cmaj.Io6ooor

\section{Standards of training}

In a recent $C M A J$ news article about the challenges currently faced by cardiac surgeons in Canada, ${ }^{1}$ Stephen Fremes, head of cardiac surgery at Sunnybrook and Women's College Health Sciences Centre in Toronto, is quoted as saying that training may need to change so that cardiac sur- 